\title{
Sु \\ Giant magnetoresistance in ferromagnet/organic semiconductor/ferromagnet heterojunctions
}

\author{
Jung-Woo Yoo, ${ }^{1,2}$ H. W. Jang, ${ }^{3}$ V. N. Prigodin,,${ }^{1}$ C. Kao, ${ }^{2}$ C. B. Eom, ${ }^{3}$ and A. J. Epstein ${ }^{1,2}$ \\ ${ }^{1}$ Department of Physics, The Ohio State University, Columbus, Ohio 43210-1117, USA \\ ${ }^{2}$ Department of Chemistry, The Ohio State University, Columbus, Ohio 43210-1173, USA \\ ${ }^{3}$ Department of Materials Science and Engineering, University of Wisconsin-Madison, Madison, Wisconsin 53706, USA
}

(Received 6 April 2009; revised manuscript received 26 October 2009; published 30 November 2009)

\begin{abstract}
We report the spin injection and transport in ferromagnet/organic semiconductor/ferromagnet (FM/OSC/FM) heterojunctions using rubrene $\left(\mathrm{C}_{42} \mathrm{H}_{28}\right)$ as an organic semiconductor spacer. For completeness of our study, both tunneling magnetoresistance (TMR) and giant magnetoresistance (GMR) were studied by varying the thickness of the rubrene layer $(5-30 \mathrm{~nm})$. A thorough study of the device characteristics reveals spin-polarized carrier injection into and subsequent transport through the OSC layer. When the thickness of the rubrene layers are beyond the tunneling limit, the device currents are limited by carrier injection and bulk transport. The carrier injection is well described with phonon-assisted field emission. The behavior of GMR in response to bias field and temperature shows significant differences from that of TMR.
\end{abstract}

DOI: 10.1103/PhysRevB.80.205207

PACS number(s): 72.25.Hg, 72.25.Dc, 73.40.Mr, 75.47.De

\section{INTRODUCTION}

The study of spin injection, transport, and dynamics in semiconductors, known as "spintronics," has received growing attention over the last decade. Observation of magnetoresistance (MR) in magnetic trilayers is a common approach to study spin injection and transport. Here, the magnetic trilayer is comprised of soft and hard magnets separated by a nonmagnetic spacer to allow parallel (on) and antiparallel (off) alignments of the magnetic layers. Then, MR, defined as $\mathrm{MR}(\%)=100 \times\left(R_{\mathrm{AP}}-R_{\mathrm{P}}\right) / R_{\mathrm{P}}$, relies on spin-dependent scattering at the interfaces and spin-polarized carrier transport in the spacer.

The spintronic applications of organic semiconductors (OSCs) were motivated by long spin lifetime in OSCs due to low spin-orbit coupling and weak hyperfine interaction. ${ }^{2-17}$ The early report of spin valve effects utilizing a thick layer $(>100 \mathrm{~nm})$ of tris (8-hydroxyquinolinato)aluminum $\left(\mathrm{Alq}_{3}\right)$ as a spacer in vertical devices ${ }^{3}$ has brought considerable attention to organic semiconductor spintronics. But, low device resistance, weak temperature $(T)$ dependence of $I-V$ curves, and low-bias $\left(V_{b}\right)$ MR have brought critiques $^{9-11}$ arguing that the reported MR (Ref. 3) originates from tunneling magnetoresistance (TMR) in locally thin areas of the OSC layer ${ }^{9,10}$ as shown for the magnetic tunnel junction using thin layers of $\mathrm{Alq}_{3}$ and rubrene $\left(\mathrm{C}_{42} \mathrm{H}_{28}\right){ }^{7,8}$ In this paper, we use the term giant magnetoresistance (GMR) referring to magnetoresistance induced by the injection and transport of spin-polarized carriers through the nonmagnetic spacers instead of tunneling through thin insulating barriers.

Organic semiconductors typically have an energy gap of more than $2 \mathrm{eV}$ (e.g., rubrene $\sim 2.3 \mathrm{eV}$; $\mathrm{Alq}_{3} \sim 2.8 \mathrm{eV}$ ) between highest occupied molecular orbital (HOMO) and lowest unoccupied molecular orbital (LUMO) levels. Thus, these materials are closer to insulators than to conventional inorganic semiconductors. The charge transport in the HOMO/ LUMO levels is provided by phonon-assisted hopping as the orbital overlap between the adjacent molecules is small and driven by the strong electric field in the organic electronic devices. In accordance with the previous criticisms ${ }^{9-11}$ con- cerning the observation of GMR, the amorphous OSC film spaced spin valve devices should have (a) extremely high junction resistance at low bias $(\sim$ several $\mathrm{mV})$ and low $T$ due to large energy gap, (b) strong $T$-dependent junction resistance due to activated carrier injection at the interface and activated carrier transport in the bulk, and (c) strong nonlinear $I-V$ curves with substantial $T$ dependence due to carrier injection and space charge effect.

Recent studies of the spin diffusion in OSC layers using muon spin rotation ${ }^{14}$ and photoemission ${ }^{15}$ techniques demonstrated spin injection into an OSC layers. However, the estimated effective spin diffusion length in OSC layers is much shorter $\left(\lambda_{s} \sim 10 \mathrm{~nm}\right)$ (Refs. 14 and 15) in comparison to the previous reports ${ }^{2,3,13}$ of the MR using " $100 \mathrm{~nm}$ " of amorphous OSC spacers. In short, the observation of GMR is still controversial and the understanding of spin injection and transport in OSC layer is still lacking due to lack of comprehensive device characteristic study. ${ }^{18}$ In this paper, we present the underlying nature of how carriers are mediated through the OSC films depending on bias, temperature, and OSC film thickness and show clear distinction between TMR and GMR and provide direct evidence of electrical spin injection and detection in OSC-spaced spin valve devices.

Though the OSC materials hold promise for their long spin lifetimes, injection and transport of spin-polarized carriers in OSC films meet significant obstacles. There are typically large band offsets between the Fermi level of typical ferromagnetic metals and the HOMO and LUMO levels of the OSCs. The dipolar barrier at the metal/OSC interface creates defect states and may interfere with spin-polarized carrier injection. ${ }^{7}$ Even when the efficient spin injection into the HOMO/LUMO levels of an OSC is achieved, hopping transport in amorphous small molecule films associated with phonon interaction may undermine efficient spin transport, especially at high $T$. Finally, there is a conductivity mismatch issue in applying highly resistive materials as the spin transporting spacer. ${ }^{19,20}$ However, the flexibility of organic chemistry has potential for significant improvement of efficient spin injection and transport in organic semiconductors. Despite the available wide range of materials and novel pro- 
(a)
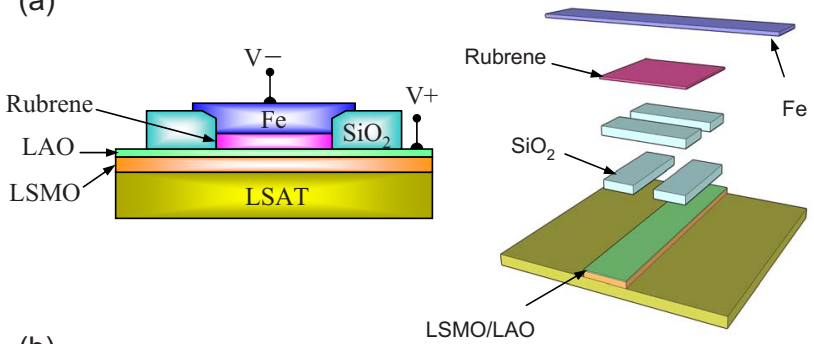

(b)

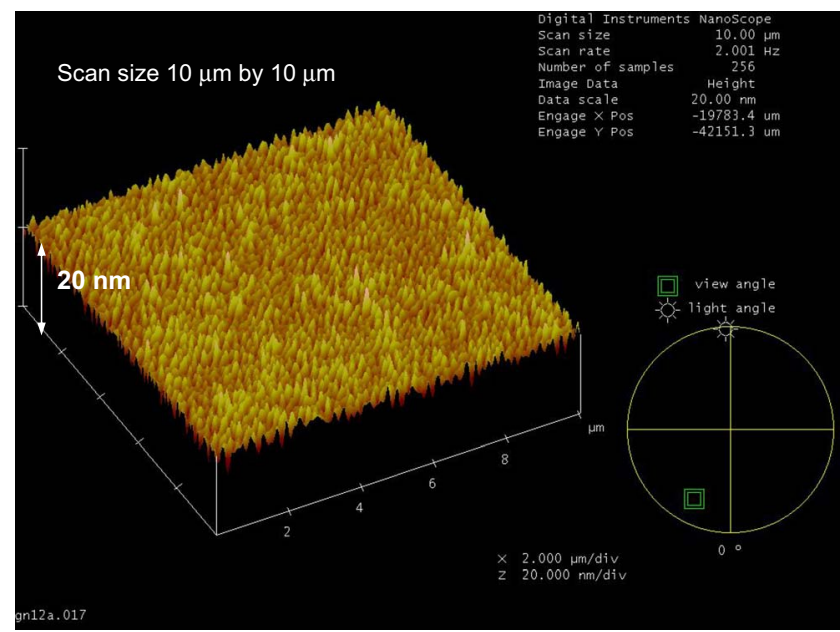

FIG. 1. (Color online) (a) The schematic view of the device structure and steps of layer depositions. (b) AFM image for $20 \mathrm{~nm}$ rubrene layer grown on (001) LAO surface.

cessing techniques applied in other organic electronics research, the spintronic applications of OSC materials have been focused on exploiting amorphous small molecule films indicating that the research in this field is still in its infancy. The evolution of this field will rely on developing desirable materials and processing techniques for the improvement of interfacial quality and efficient spin diffusion in organic spacers.

In this work, we fabricated spin valve structures [Fig. 1(a)] using $\mathrm{La}_{2 / 3} \mathrm{Sr}_{1 / 3} \mathrm{MnO}_{3}$ (LSMO) and $\mathrm{Fe}$ as an anode and a cathode, respectively, and rubrene as the OSC spacer. To improve interfacial quality between the LSMO and rubrene, we applied a thin layer $(1.2 \mathrm{~nm})$ of $\mathrm{LaAlO}_{3}$ (LAO). The observed TMR in magnetic tunnel junction using a thin rubrene layer $(5 \mathrm{~nm})$ displays spin-conserved tunneling through the hybrid barrier $[\operatorname{LAO}(1.2 \mathrm{~nm}) / \operatorname{rubrene}(5 \mathrm{~nm})]$, as reported by Shim et al. ${ }^{8}$ The $T$ - and $V_{b}$-dependent behavior of TMR in our devices is similar to the previously reported GMR behavior in organic-based spin valves using LSMO, , 13 in agreement with a early suggestion by $\mathrm{Xu}$ et al. ${ }^{9}$ As the thickness of the rubrene layer increases, devices are limited by carrier injection and transport. For intermediate thickness $(d \sim 20 \mathrm{~nm})$, the carrier injection described by thermionic field emission controls the device current. Applying high bias is needed to have viable device current as well as to observe MR. The observed GMR for the devices with intermediate thickness of rubrene layer shows spin-polarized carrier injection into, and subsequent transport through the rubrene layer. The responses of GMR in our devices to bias field and $T$ show clear differences from that of TMR through the thin hybrid (oxide/OSC) layer.

This paper is organized as follows. In the following Sec. II, we present our device fabrication procedure. We then provide background for tunneling vs carrier injection and transport in organic spin valves. The variation in MR in thin hybrid barrier device in response to temperature and bias field is presented in Sec. IV. In Sec. V, we display $T$ - and $V_{b}$-dependent characteristics in our thick rubrene spaced devices. We analyze these experimental results in terms of phonon-assisted field emission. We then present the temperature and bias field dependences of MRs in our thick rubrene spaced devices in Sec. VI. Finally, Sec. VII is for the summary and conclusions.

\section{DEVICE FABRICATION}

Figure 1(a) shows a schematic view of our device structure and illustrates the layer deposition steps. The epitaxial $50 \mathrm{~nm}$ of LSMO on lattice matching (001) $\left(\mathrm{LaAlO}_{3}\right)_{0.3}\left(\mathrm{Sr}_{2} \mathrm{AlTaO}_{6}\right)_{0.7}(\mathrm{LSAT})$ substrate was grown by pulsed laser deposition and capped with three unit cells of epitaxial LAO $(1.2 \mathrm{~nm})$. The purposes of using the thin LAO capping layer are (a) to improve metal/OSC interface by preventing chemical reaction between small molecule and metal at the interface, ${ }^{7,8}$ (b) to promote efficient spin-polarized carrier emission at the interface, ${ }^{21}$ and (c) to provide superior protection of LSMO surface polarization than $\mathrm{SrTiO}_{3}$ does. ${ }^{22}$ $30 \mathrm{~nm}$ of $\mathrm{Fe}$ was used for the second ferromagnet and deposited by e-beam. The junction area is $0.2 \times 0.2 \mathrm{~mm}^{2}$, which is enclosed by 300-nm-thick $\mathrm{SiO}_{2}$ layer deposited by e-beam using a shadow mask [Fig. 1(a)]. The purpose of $\mathrm{SiO}_{2}$ layer is to cover the edges of the junction area. The LSMO electrode is already $50 \mathrm{~nm}$ thick making it difficult to achieve a well-defined junction area for thin rubrene layered devices.

The sublimed grade rubrene (Aldrich) was used for OSC films. The rubrene layers were deposited with controlled source temperature $\left(\sim 105^{\circ} \mathrm{C}\right)$ with a deposition rate of $\sim 2.5 \mathrm{~nm} / \mathrm{min}$ at base pressures $\sim 10^{-7}$ mbar. Figure 1(c) shows atomic force microscopy images of $20 \mathrm{~nm}$ rubrene layers deposited on (001) LAO surface. The typical rms roughness of rubrene films were around $1 \mathrm{~nm}$. The thickness of the rubrene layers was determined by a quartz crystal monitor and verified with a Dektak profilometer. We studied both the TMR and GMR with varying thickness of the rubrene layer from tunneling limit $(d \sim 5 \mathrm{~nm})$ to carrier injection and transport limit $(d \geq 20 \mathrm{~nm})$. Though our thermally deposited rubrene layer displays relatively homogeneous surface morphology, its typical rms roughness of $1 \mathrm{~nm}$ suggests that the actual charge transport distance of a rubrene layer would be slightly shorter than the determined film thickness. Also, roughness of our rubrene layer suggests possible presence of pinhole channels for $5 \mathrm{~nm}$ rubrene layers and prevents us from performing precise study on the $\mathrm{nm}$ scale thickness dependence of the tunneling current.

\section{TUNNELING VS CARRIER INJECTION AND TRANSPORT}

Figure 2 displays a schematic view of tunneling vs carrier injection for OSC-spaced magnetic heterojunction devices. 
(a)

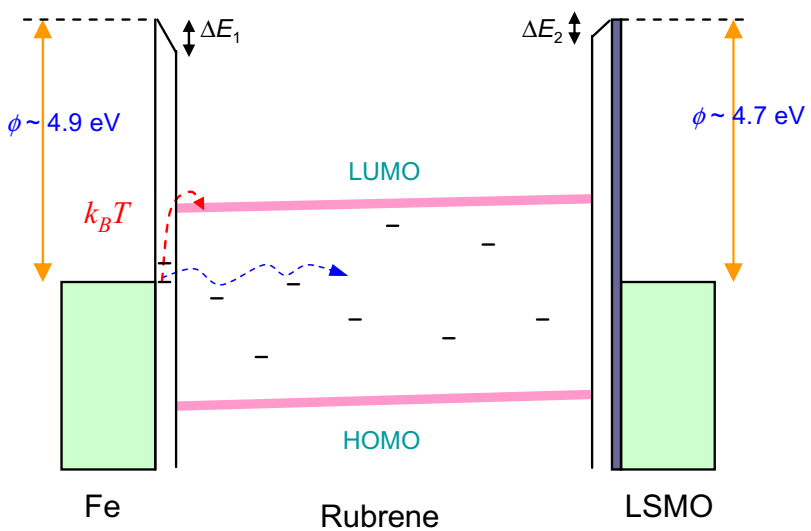

Low bias

(b)

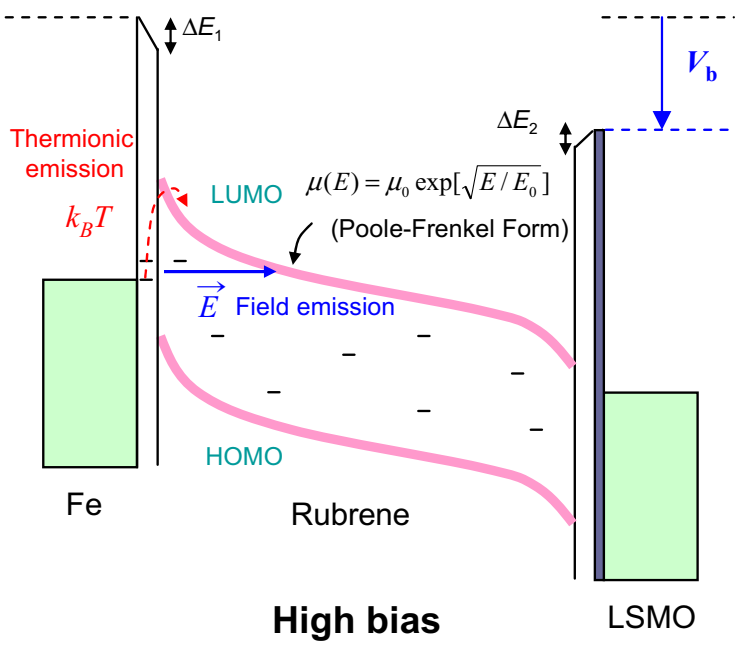

FIG. 2. (Color online) Tunneling vs carrier injection and transport. (a) Schematic illustration of device operation at low bias. When the thickness of rubrene layers is relatively thin, device currents at low bias are provided by tunneling through the defect states in the energy gap. $\Delta E_{1}$ and $\Delta E_{2}$ describe the modification of level alignment at the metal/OSC interfaces due to dipolar barriers (Ref. 23). (b) The schematic view of device operation at high bias. The thermionic field emission at the metal/OSC interface controls the carrier injection into either HOMO/LUMO levels of OSC layers.

We describe the device current as a sum of two distinct pathways, one is tunneling through the optical gap and the other is carrier injection and transport through the HOMO and/or LUMO levels. The elastic tunneling through the barrier is generally considered to be limited to $2-3 \mathrm{~nm}$ of the insulator. ${ }^{24}$ Here, we refer the tunneling through the OSC layer for both the direct tunneling through the OSC layer and the multistep tunneling through the defect states in the energy gap of the OSC materials. At low bias ( several $\mathrm{mV})$, the tunneling current through the defect states in the optical gap serves as a main channel of device current. These defect states are rare and do not provide continuous charge transport pathway. Carriers in these defect states can be elevated via strong electric field and/or thermionic emission to the more dense HOMO/LUMO levels, where electron/holes can more easily hop among energy levels of adjacent molecules.
At low bias and low $T$, tunneling current diminishes as the thickness of the rubrene layer is increased.

With increasing thickness of the rubrene layer, the device current will be controlled by the carrier injection and transport into/in the OSC layer. Figure 2(b) shows schematic description of carrier injection into either HOMO or LUMO levels of OSC layer by applying high bias. Injection into HOMO/LUMO can be mediated by phonons, especially at high temperatures. This thermionic emission decreases as the temperature is lowered. At very low $T$, field emission dominates the device currents. The thermionic field emission at the interfaces, together with phonon-assisted hopping in HOMO/LUMO levels will introduce strong $T$-dependent device current reflecting thermal activation.

Figure 3(a) displays $I-V$ characteristics of LSMO/LAO/ rubrene/Fe devices with 5-, 20-, 30-, and 50-nm-thick rubrene layers showing strong nonlinearity at $10 \mathrm{~K}$. As the thickness of rubrene layer is increased, the device currents are strongly limited by injection. The dc device resistance, which is determined by linear fit at low bias region (typically up to $10 \mathrm{mV}$ ) is very high for devices with thick rubrene layers $(d \geq 20 \mathrm{~nm})$. When the thickness of the rubrene layer beyond the tunneling limit, the device currents are supplied by thermionic emission at high $T$ with subsequent phononassisted hopping in the HOMO/LUMO levels and exponentially decreases as $T$ is lowered [Fig. 3(b)]. Finally, at low $T$, only tunneling through rare defect states accounts for the device current and is nearly negligible for the thick OSCspaced spin valves. Typical device resistances at low bias for device with $d \geq 50 \mathrm{~nm}$ thick rubrene layer exceed instrumental limits $(\sim G \Omega)$ below $100 \mathrm{~K}$. This is in contrast to previously reported low-resistance MR at low bias and low $T$ in organic-based spin valve using thick OSC layers $(\sim 100 \mathrm{~nm})$. Our study suggests that one has to be careful in assessing determined fundamental properties, such as spinrelaxation time and diffusion length derived from low-bias $(\sim$ several $\mathrm{mV})$ measurements with thick OSC layers. ${ }^{3,6}$

Figure 3(c) shows $T$-dependent resistance for 5, 20, and $50 \mathrm{~nm}$ rubrene layer device and LSMO electrode. For a thin rubrene layer, the sufficient current is supported by tunneling through defect states over all $T$. Since LSMO film also has strong $T$ dependence, it is not plausible to interpret $T$-dependent device current for thin rubrene layered devices $(d=5 \mathrm{~nm})$. However, one can still deduce that the effective junction resistance for $5 \mathrm{~nm}$ device increases by approximately twofold as $T$ is lowered from 300 to $10 \mathrm{~K}$ by subtracting the resistance of LSMO electrode. This $T$-dependent change in device resistance for our hybrid barrier $[\mathrm{LAO}(1.2$ $\mathrm{nm}) /$ rubrene $(5 \mathrm{~nm})]$ is similar to the previous report for the hybrid barrier of $\mathrm{Al}_{2} \mathrm{O}_{3}(0.5 \mathrm{~nm}) /$ rubrene $(5-6 \mathrm{~nm}){ }^{8}$ This suggests tunneling through the hybrid barrier (LAO/rubrene) largely accounts for the $5 \mathrm{~nm}$ rubrene device currents, although currents through pinhole channels likely contribute to the total device currents. Also, using a double barrier (LAO and rubrene) alleviates the effect of pinholes in our thin (5 $\mathrm{nm})$ OSC-spaced devices.

As the thickness of rubrene layer increases beyond the tunneling limit, carrier injection by thermionic and/or bias field emission and subsequent field-driven transport in the OSC layer control device currents. The device current for 

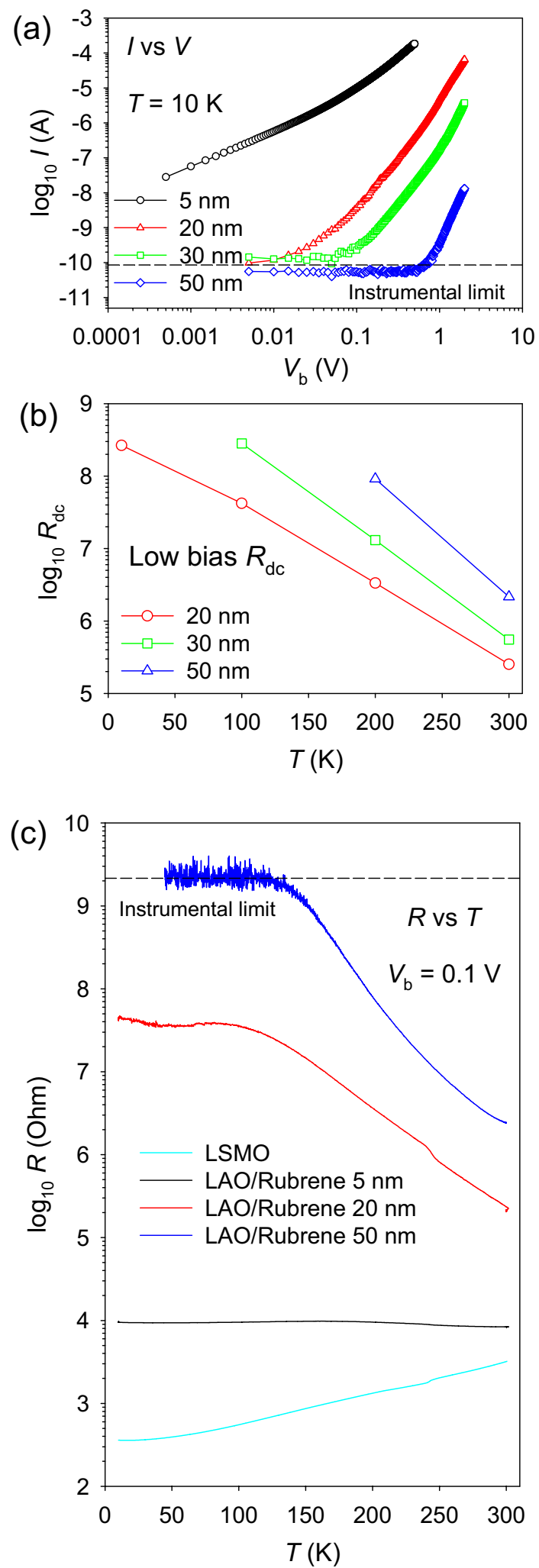

FIG. 3. (Color online) (a) $I-V$ curves of LSMO $(50 \mathrm{~nm}) / \mathrm{LAO}$ $(1.2 \mathrm{~nm}) /$ Rubrene/Fe $(30 \mathrm{~nm})$ devices for the thickness of rubrene layer $d=5,20,30$, and $50 \mathrm{~nm}$ at $10 \mathrm{~K}$. Devices are injection limited as $d$ increases beyond tunneling limit. (b) Temperature dependence of low bias ( $\sim$ several $\mathrm{mV}) R_{\mathrm{dc}}$ showing exponential increase as $T$ is lowered. (c) Temperature dependence of resistance for LSMO electrode and 5-, 20-, and 50-nm-thick rubrene devices at applied bias of $0.1 \mathrm{~V}$. thick OSC layers is strongly $T$ dependent reflecting activation processes and shows clear distinction from that of thin rubrene layered devices [Fig. 3(c)]. Further detailed study on the effect of thermionic field emission will be discussed in the section of phonon-assisted field emission.

\section{TUNNELING MAGNETORESISTANCE IN FM/OSC/FM HETEROJUNCTIONS}

Figure 4(a) displays $I-V$ and $d I / d V$ curves of a $5 \mathrm{~nm}$ rubrene device $[\operatorname{LSMO}(50 \mathrm{~nm}) / \mathrm{LAO}(1.2 \mathrm{~nm}) /$ Rubrene $(5$ $\mathrm{nm}) / \mathrm{Fe}(30 \mathrm{~nm})]$ at $10 \mathrm{~K}$. The $d I / d V$ was measured by the standard lock-in technique. As discussed in previous literature, ${ }^{7}$ the absence of a zero bias dip in the $d I / d V$ curve indicates that hybrid barrier (LAO/rubrene) forms a welldefined tunneling barrier. Figure 4(b) displays magnetization $(M)$ vs in-plane magnetic field $(H)$ at $10 \mathrm{~K}$ for individual $\mathrm{Fe}$ $(30 \mathrm{~nm})$ and LSMO [50 $\mathrm{nm}$ on (001) LSAT] films recorded with a superconducting quantum interference device (SQUID) magnetometer. MR curves of a $5 \mathrm{~nm}$ rubrene device for $V_{b}=10 \mathrm{mV}$ and in-plane $H$ at different $T(10,50$, 100 , and $150 \mathrm{~K}$ ) are displayed in Fig. 4(c). The steps of the MR curves at each $T$ well correspond to the $T$-dependent in-plane coercivity $\left(H_{c}\right)$ for Fe and LSMO electrodes, as shown in Fig. 4(d). The coercivity in Fig. 4(d) is determined from the individual $\mathrm{Fe}(30 \mathrm{~nm})$ and LSMO $(50 \mathrm{~nm})$ electrodes using a SQUID magnetometer. The substantial decrease in MR as $T$ is increased [see Fig. 6(d)] is attributed to the $T$-dependent surface polarization of the LSMO electrode. ${ }^{25}$ Similar $T$ dependence of MR was commonly reported for various LSMO-based trilayers ${ }^{26}$ as well as organic-based spin valve using LSMO electrodes. ${ }^{3,9,13,27,28}$

The bias dependence of MR curves for a typical $5 \mathrm{~nm}$ rubrene device at $10 \mathrm{~K}$ is presented in Fig. 4(e) for positive biases and (f) for negative biases. Our TMR device exhibits positive MR over all biases. The steps of MR curves for all biases show excellent correspondence to the coercivities of Fe $(30 \mathrm{~nm})$ and LSMO $(50 \mathrm{~nm})$ electrodes. The MR vs $V_{b}$ in Fig. 4(g) was determined by the comparison of $I-V$ curves for parallel (at $H=500 \mathrm{Oe}$ ) and antiparallel (at $H=$ $-150 \mathrm{Oe}$ ) configurations of magnetic layers. The asymmetric MR for $V_{b}$ is due to different electrodes (Fe, LSMO) and is similar to previous observations. $3,9,27$

The MR of our devices is positive over all $T$ and $V_{b}$ in contrast to previous reports, which showed negative MR in organic-based spin valves. ., $^{3,13,27}$ The difference between our device and previously reported organic spin valves is that we used $\mathrm{Fe}$ instead of $\mathrm{Co}$ for the second ferromagnetic layer. Another possible origin of the negative MR in the spin valves $^{3,9,13,27}$ is the presence of pinhole channels. The ballistic current through nanoscale metallic contact could induce inversion from positive to negative $\mathrm{MR}$ as suggested theoretically ${ }^{29}$ and experimentally observed in LSMO-based trilayers. ${ }^{30}$

\section{PHONON-ASSISTED FIELD EMISSION}

With increasing thickness of rubrene layer, devices display stronger nonlinear $I-V$ curves and become injection lim- 
(a)

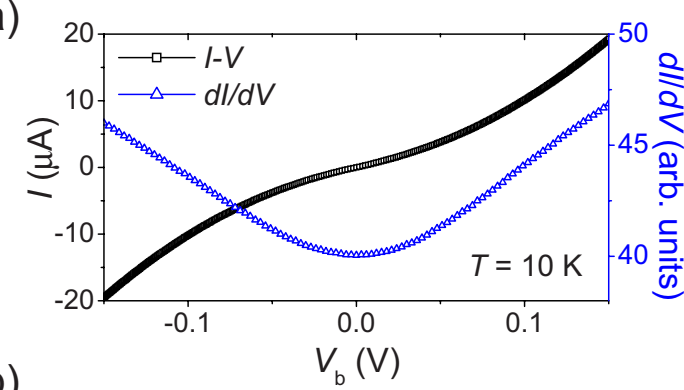

(b)
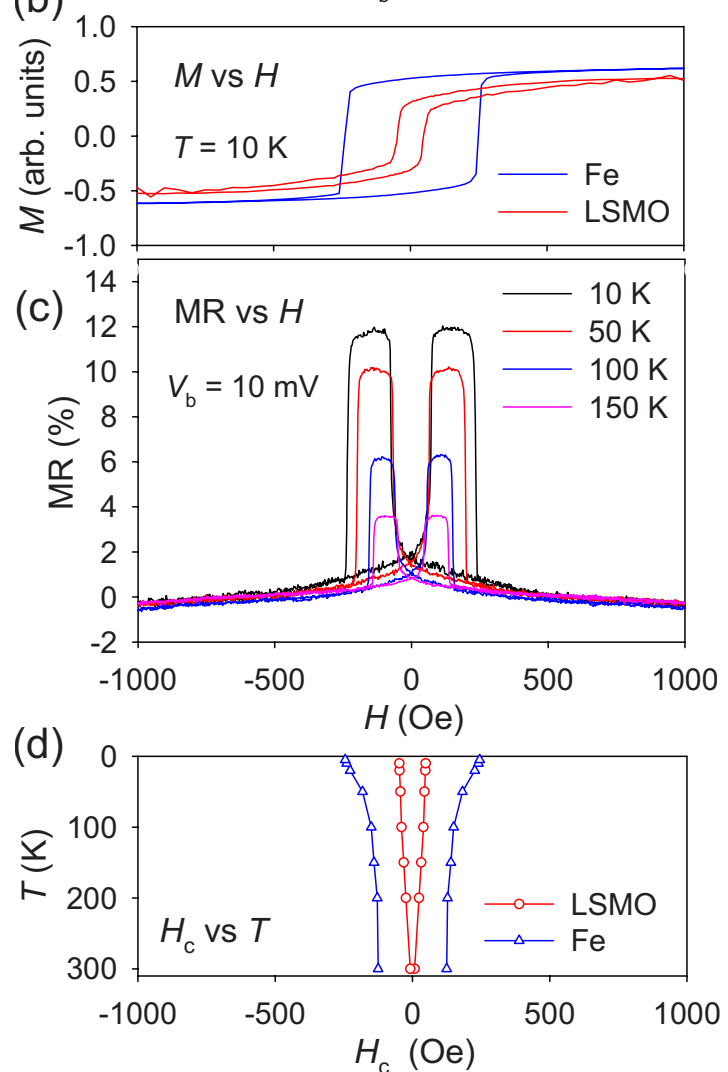

(e)

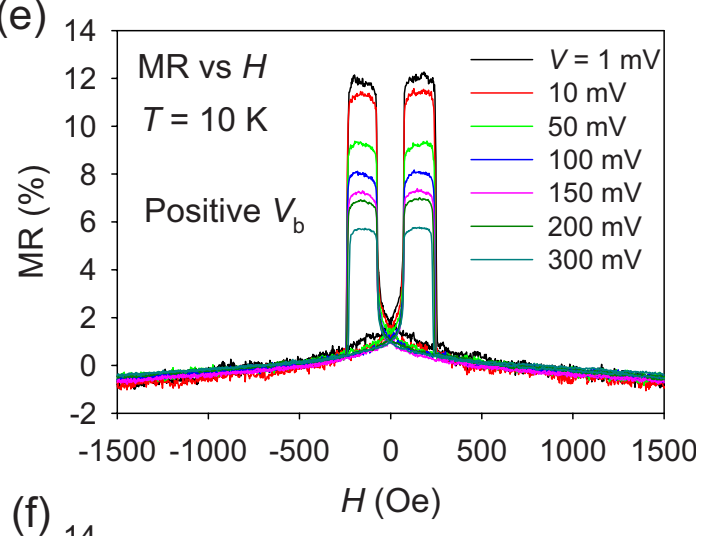

(f)

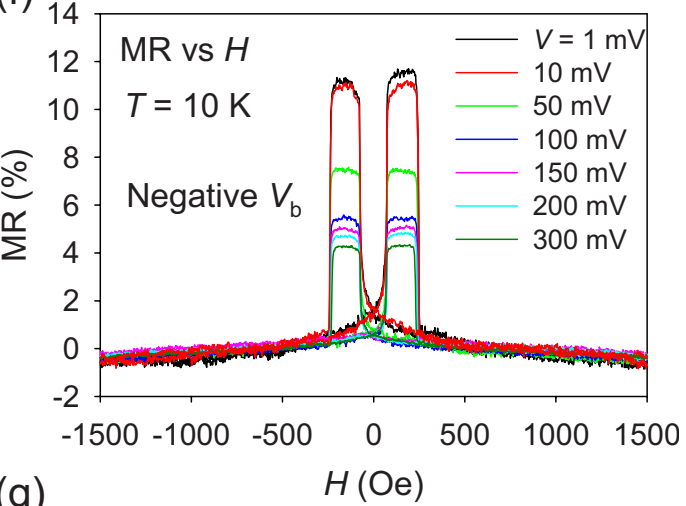

(g)

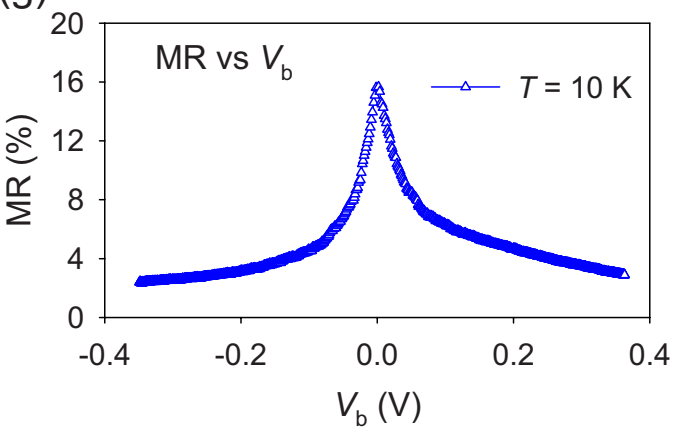

FIG. 4. (Color online) Tunneling magnetoresistance of the hybrid magnetic tunnel junction. (a) $I-V$ and $d I / d V$ curves for magnetic tunnel junction of $5 \mathrm{~nm}$ rubrene device $[\mathrm{LSMO}(50 \mathrm{~nm}) / \mathrm{LAO}(1.2 \mathrm{~nm}) /$ rubrene $(5 \mathrm{~nm}) / \mathrm{Fe}(30 \mathrm{~nm})]$. (b) $M$ vs $H$ at $10 \mathrm{~K}$ for Fe $(30 \mathrm{~nm})$ and LSMO $(50 \mathrm{~nm})$ on LSAT(001) substrate. (c) MR curves of $5 \mathrm{~nm}$ rubrene device at $T=10,50,100$, and $150 \mathrm{~K}$. (d) $H_{c}$ vs $T$ for Fe (30 nm) and LSMO $(50 \mathrm{~nm})$ magnetic layers recorded by SQUID. (e) Positive-bias dependence of MR curves for $5 \mathrm{~nm}$ rubrene device at $10 \mathrm{~K}$. (f) Negative-bias dependence of MR curves for $5 \mathrm{~nm}$ rubrene device at $10 \mathrm{~K}$. (g) The magnitude of MR as a function of $V_{b}$ for $5 \mathrm{~nm}$ rubrene device. MR vs $V_{b}$ of $5 \mathrm{~nm}$ rubrene device at $10 \mathrm{~K}$ is determined by the difference in $I-V$ curves between parallel $(H=500$ Oe $)$ and antiparallel $(H=$ $-150 \mathrm{Oe}$ ) configurations for the $5 \mathrm{~nm}$ rubrene device.

ited as shown in Figs. 5(a) and 5(b) for 20 and $50 \mathrm{~nm}$ rubrene devices, respectively. Figures 5(c) and 5(d) show $\log I$ vs $\log V$ plots for 20 and $50 \mathrm{~nm}$ rubrene devices. As the bias field is increased, all curves for different $T$ asymptote to the same slope, which is a typical characteristic for charge injection via field emission at metal/organic interface. Figures 5(e) and 5(f) display $T$-dependent device resistances for 20 and $50 \mathrm{~nm}$ rubrene devices, respectively. The noise at low $V_{b}$ and low $T$ for $50 \mathrm{~nm}$ rubrene device is due to our instrumental limit. The substantial increase in device current as $T$ increases over $100 \mathrm{~K}$ can be attributed to active thermionic emission at the interface, once the device is limited by injection. A similar $T$ dependence of device conductance was previously reported for an organic spin valve with a thin rubrene layer. $^{8}$ The LSMO resistance strongly decreases as $T$ is lowered ${ }^{2,28}$ but this resistance can be ignored as it is substantially smaller than the total device resistance for thick rubrene layered devices $(d \geq 20 \mathrm{~nm})$ [see Fig. 3(c)].

We describe the carrier injection in our spin valve devices with thermionic field emission at the metal/OSC interface as it was discussed in Sec. III. We adopt the theoretical model developed by Kiveris and Pipinys, ${ }^{31,32}$ which accounts for multiphonon activation of electrons together with field emission from defect states at the interfaces to the conduction band for the metal/semiconductor junction. The phononassisted tunneling rate of electrons under the electric field at the metal/semiconductor interface is as follows: ${ }^{31,32}$ 
(a)
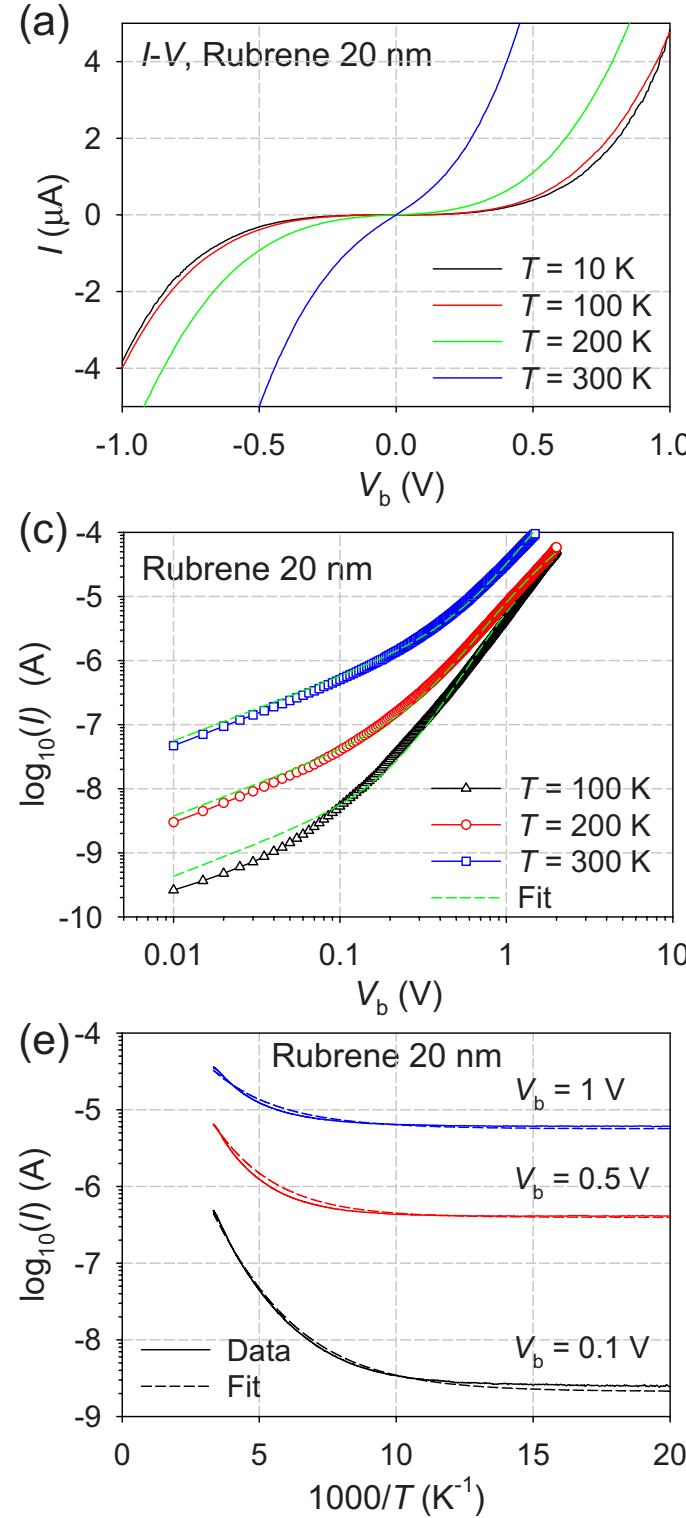
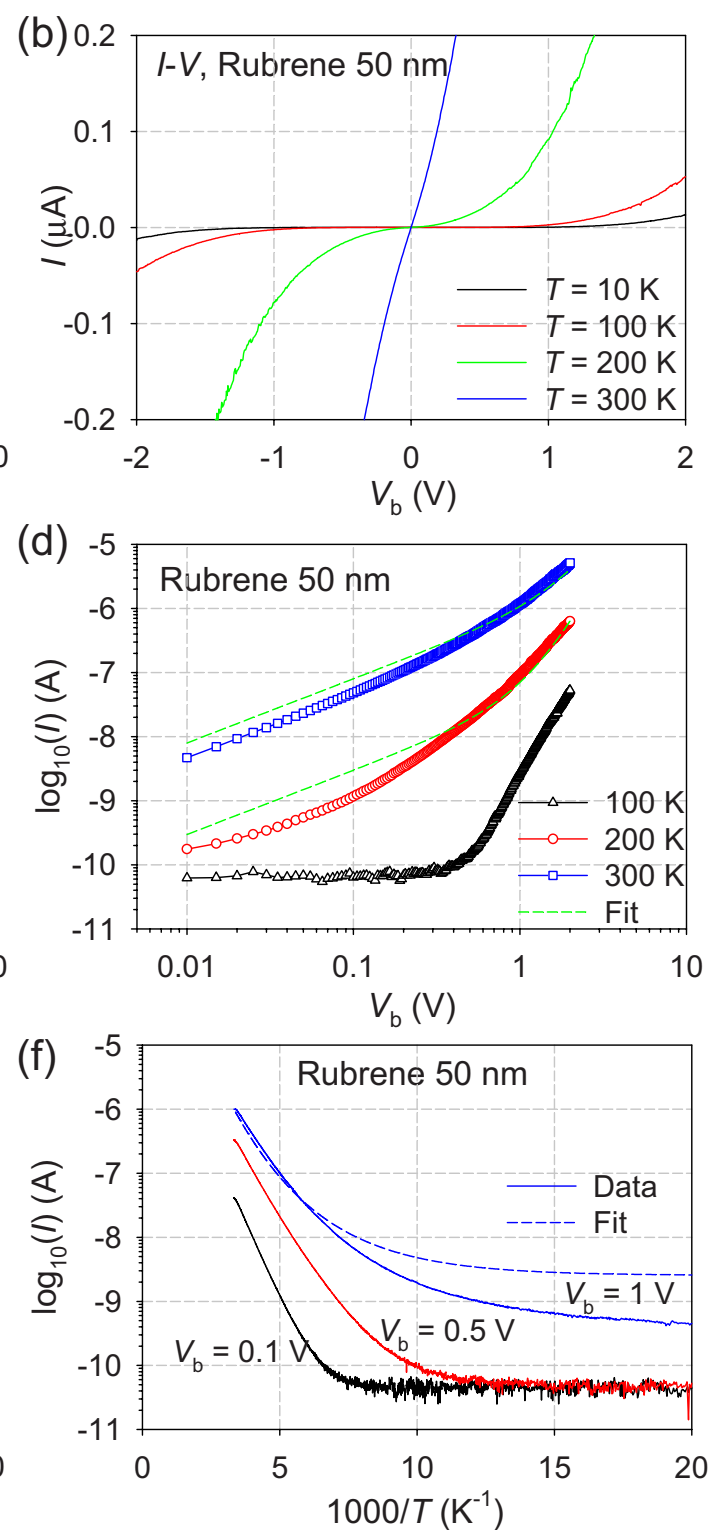

FIG. 5. (Color online) Device characteristics of the organic spin valves. (a) and (b) $T$ dependence of $I$ - $V$ curves for 20 and $50 \mathrm{~nm}$ rubrene devices. (c) and (d) $\log I$ vs $\log V$ plots for 20 and $50 \mathrm{~nm}$ rubrene devices at $T=100,200$, and $300 \mathrm{~K}$. (e) and (f) $I$ vs $1000 / T$ for 20 and 50 $\mathrm{nm}$ rubrene devices at $V_{b}=0.1,0.5$, and $1 \mathrm{~V}$. Dashed lines in (c)-(f) are fits to the Eq. (1) with fixed $a=1.4, \hbar \omega=0.034 \mathrm{eV}, m^{*}=1.2 m_{e}$, and slight adjustment of $\varepsilon_{T}=0.67 \pm 0.01 \mathrm{eV}$ for each plot. $\varepsilon_{T}=0.660$ [for $100 \mathrm{~K}$ in (c)], 0.666 [for $200 \mathrm{~K}$ in (c)], 0.674 [for $300 \mathrm{~K}$ in (c)], 0.673 [for $200 \mathrm{~K}$ in (d)], 0.662 [for $300 \mathrm{~K}$ in (d)], 0.678 [for $0.1 \mathrm{~V}$ in (e)], 0.674 [for $0.5 \mathrm{~V}$ in (e)], 0.660 [for $1 \mathrm{~V}$ in (e)], and 0.676 [for $1 \mathrm{~V}$ in (f)].

$$
\begin{aligned}
W_{T}= & \frac{e E}{\left(8 m^{*} \varepsilon_{T}\right)^{1 / 2}}\left[\left(1+\gamma^{2}\right)^{1 / 2}-\gamma\right]^{1 / 2}\left[1+\gamma^{2}\right]^{-1 / 4} \\
& \times \exp \left\{-\frac{4}{3} \frac{\left(2 m^{*}\right)^{1 / 2}}{e E \hbar} \varepsilon_{T}^{3 / 2}\left[\left(1+\gamma^{2}\right)^{1 / 2}-\gamma\right]^{2}\right. \\
& \left.\times\left[\left(1+\gamma^{2}\right)^{1 / 2}+\frac{1}{2} \gamma\right]\right\},
\end{aligned}
$$

where

$$
\gamma=\frac{\left(2 m^{*}\right)^{1 / 2} \Gamma^{2}}{8 e \hbar E \varepsilon_{T}^{1 / 2}} .
$$

Here $\varepsilon_{T}$ is the energetic depth of defect states, $E=V_{b} / d$ is the applied electric field, $\Gamma^{2}=8 a(\hbar \omega)^{2}(2 n+1)$ is the width of the defect states broadened by the optical phonons, $n$ $=1 /\left[\exp \left(\hbar \omega / k_{\mathrm{B}} T\right)-1\right]$, and $a$ is the electron-phonon coupling constant $\left[a=\Gamma_{0}^{2} / 8(\hbar \omega)^{2}\right]$. For the $20 \mathrm{~nm}$ rubrene device, qualitatively good fits can be achieved over all $T$ and $V_{b}$ with fixed parameters of $a=1.4, \hbar \omega=0.034 \mathrm{eV}, m^{*}=1.2 m_{e}$ and slight adjustment of $\varepsilon_{T}=0.67 \pm 0.01 \mathrm{eV}$ for each plot in Figs. 5(c) and 5(e). The results show that $20 \mathrm{~nm}$ rubrene 
(a)

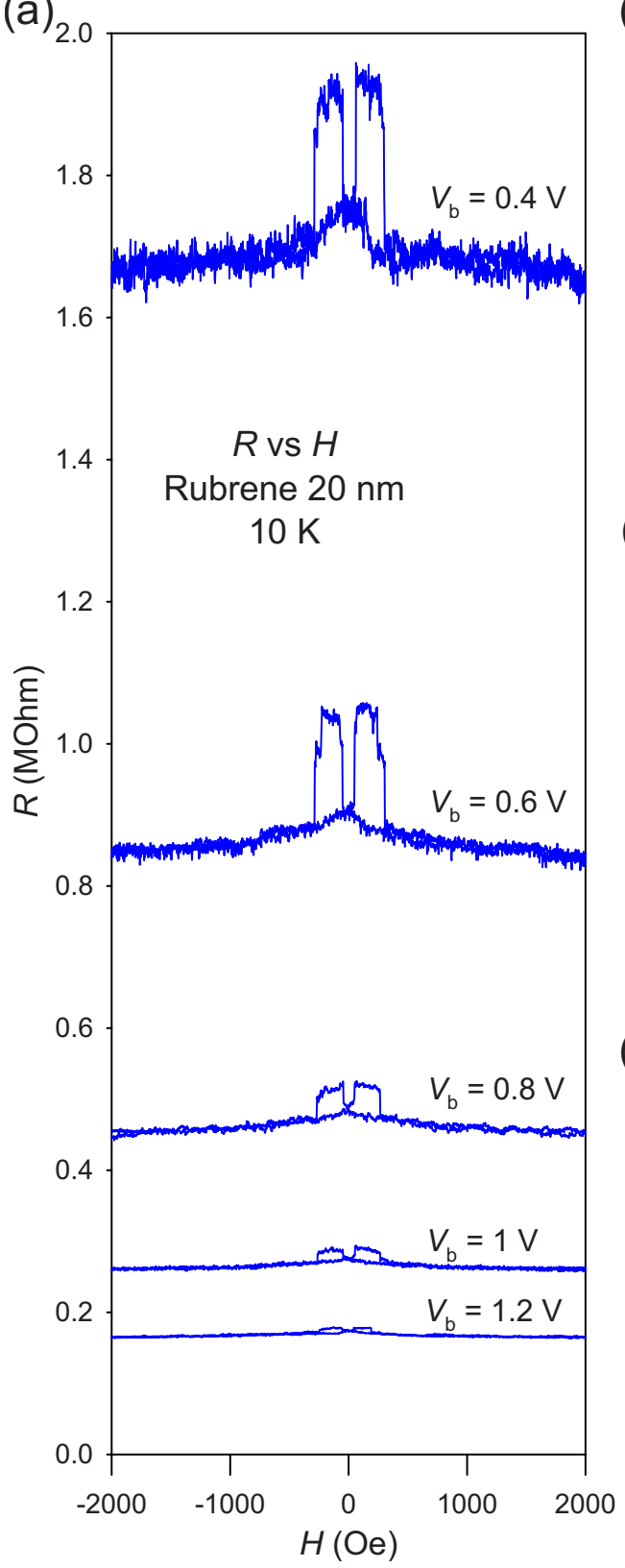

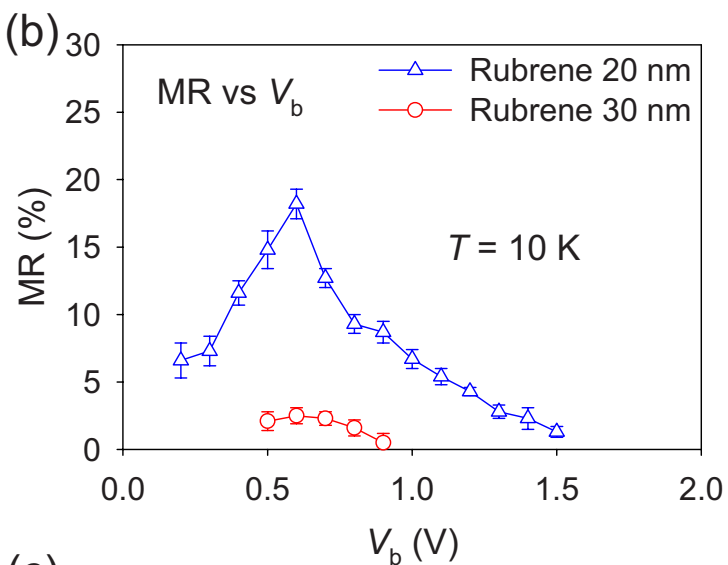
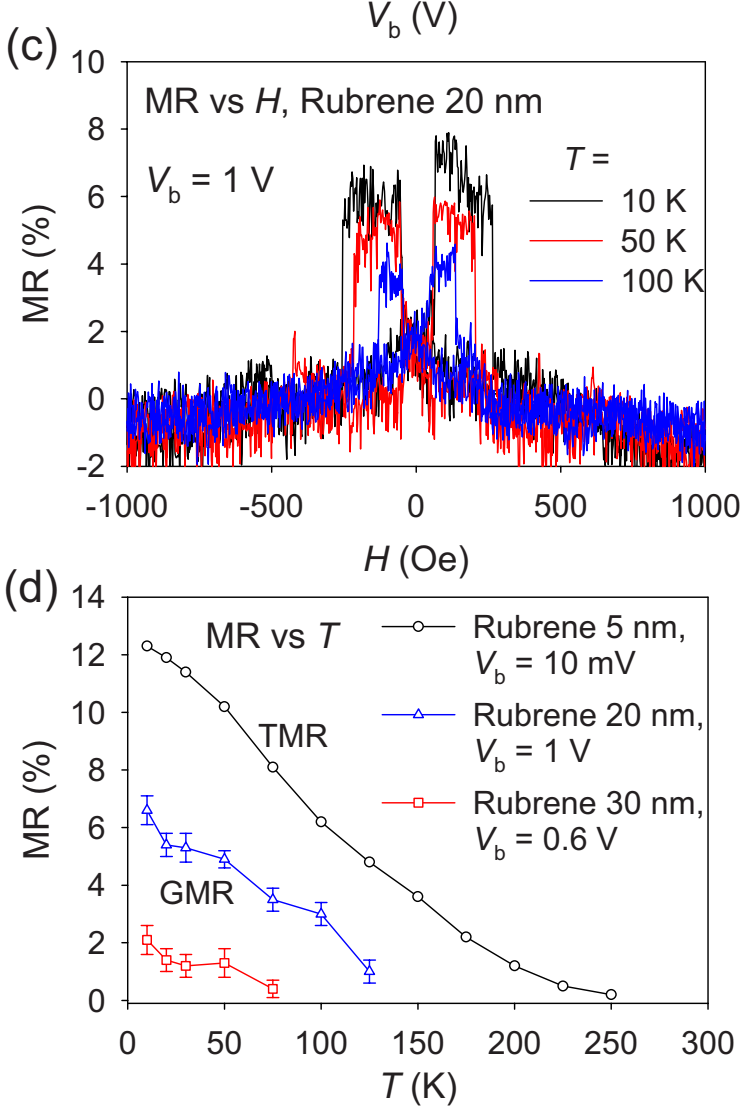

FIG. 6. (Color online) Giant magnetoresistance of the organic spin valves. (a) MR curves for $20 \mathrm{~nm}$ rubrene device for a different $V_{b}$ at $10 \mathrm{~K}$. Device resistance decreases as $V_{b}$ increases showing strong nonlinearity of $I$ - $V$ characteristic. (b) MR vs $V_{b}$ plot for 20 and $30 \mathrm{~nm}$ rubrene devices at $10 \mathrm{~K}$. (c) MR curves of $20 \mathrm{~nm}$ rubrene device for different $T$ at $V_{b}=1 \mathrm{~V}$. The steps of MR well correspond to the $H_{c}$ of ferromagnetic layers at all $T$. (d) Comparison of $T$ dependence of MR between TMR ( $5 \mathrm{~nm}$ rubrene device) and GMR (20 and $30 \mathrm{~nm}$ rubrene devices).

device currents are controlled by the carrier injection. MR of this device relies on the spin-dependent carrier injection at the interface. However, the phonon-assisted tunneling, which becomes effective as $T$ increases over $100 \mathrm{~K}$, would affect on the spin polarization of injected carriers.

Once the carriers are injected into an OSC layer, the mobility of electrons and holes also strongly rely on the applied electric field, for example, $\mu \sim \mu_{0} \exp \sqrt{E / E_{0}}$ (Poole-Frenkel form). Therefore, the slight deviation of the fitting at low bias in Fig. 5(c) likely originates from the bulk resistance of OSC, which becomes larger as $T$ is lowered. As the thickness of the rubrene layer is increased further, the carrier transport in the bulk controls the device current. A larger deviation for our fitting to $50 \mathrm{~nm}$ rubrene device currents can be observed at low bias and low $T$ as shown in Figs. 5(d) and 5(f). Fitting for $50 \mathrm{~nm}$ rubrene device was performed with the same fixed parameters of $a=1.4, \hbar \omega=0.034 \mathrm{eV}, m^{*}=1.2 m_{e}$ and a slight adjustment of $\varepsilon_{T}=0.67 \pm 0.01 \mathrm{eV}$ for each plot. Unlike interfacial barrier resistance, which nearly saturates below 100 $\mathrm{K}$ for the relatively high bias, the phonon-assisted hopping conductance in the OSC layer continues to decrease to low $T$ following an exponential $T$ dependence. This introduces a strong deviation of our fit to temperature-dependent device resistance for $50 \mathrm{~nm}$ rubrene layer as shown in Fig. 5(f) for 
$T<100 \mathrm{~K}$. In sum, for the thicker rubrene layer device $(d$ $\geq 50 \mathrm{~nm}$ ), higher bias needs to be applied for efficient field emission as well as for supporting drift current (spacecharge-limited regime, $I \propto V^{2} / d^{3}$ ). Hopping transport over long distances and dephasing of charge-carrier spins in the bulk reduces the spin polarization of carriers.

\section{GIANT MAGNETORESISTANCE IN FM/OSC/FM HETEROJUNCTIONS}

Figure 6(a) displays MR curves for different $V_{b}$ at $10 \mathrm{~K}$ for $20 \mathrm{~nm}$ rubrene device, which was presented in Fig. 5 . Resistance of $20 \mathrm{~nm}$ rubrene devices at low-bias and low $T$ is extremely high due to negligible carrier injection, which is in contrast to previously reported low-bias and low-resistance GMR results at low $T$ for thick layer of OSC spacer. ${ }^{3,6}$ For the $20 \mathrm{~nm}$ rubrene device, clear positive MR signals were detected from $200 \mathrm{mV}$ of $V_{b}$. An increase in MR was observed up to $600 \mathrm{mV}$, then MR decreased as $V_{b}$ increased further [Fig. 6(b)]. For the $30 \mathrm{~nm}$ rubrene device, MR was detectable when the $V_{b}$ was increased up to $500 \mathrm{mV}$ then became negligible when $V_{b}$ increased to $1 \mathrm{~V}$ [Fig. 6(b)]. No MR was observed for 40 and $50 \mathrm{~nm}$ rubrene devices over all $T$ and $V_{b}$ (up to several $\mathrm{V}$ ). We propose the absence of MR for thick rubrene layers is because the channel distance is longer than the spin-diffusion length $\left(\lambda_{s}\right)$ in amorphous rubrene layer, as viable device current is still measurable as long as high bias is applied.

MR curves of $20 \mathrm{~nm}$ rubrene device for different $T$ at $V_{b}=1 \mathrm{~V}$ are presented in Fig. 6(c). The steps of MR curves at different $T$ well correspond to the $H_{c}$ of Fe and LSMO as presented in Fig. 4(d). This excludes the possibility that the magnetoresistance in our device is induced by ferromagnetic Fe cluster embedded in OSC layer as proposed by Vinzelberg et al. ${ }^{11}$ The $T$ dependence of MR for 20 and $30 \mathrm{~nm}$ rubrene devices are presented in Fig. 6(d) and compared with $T$ dependence of TMR for $5 \mathrm{~nm}$ rubene device. No clear MR was observed above $150 \mathrm{~K}$ for the $20 \mathrm{~nm}$ rubrene device. Rapid drop in MR as $T$ is increased above $100 \mathrm{~K}$ for the $20 \mathrm{~nm}$ rubrene device can be associated with the thermionic emis- sion at the interface of the device. For the $30 \mathrm{~nm}$ rubrene device, the MR disappears above $75 \mathrm{~K}$. The absence of MR for thicker rubrene devices $(d>30 \mathrm{~nm})$ could be attributed to hopping transport and thermionic emission, especially at high $T$.

\section{SUMMARY AND CONCLUSIONS}

In summary, we presented both TMR and GMR with an OSC spacer and their very different behaviors in response to $V_{b}$ and $T$. For thick layer of rubrene spacer, the devices are limited by carrier injection and transport. For the intermediate thickness $(d \sim 20 \mathrm{~nm})$, the carrier injection controls the device current. The GMR for intermediate thickness shows strong $V_{b}$ and $T$ dependence as a result of the activation processes of carrier injection and transport in amorphous OSC layer, in contrast to previous reports ${ }^{3,13}$ and recent theoretical study. ${ }^{33}$ For a thicker OSC layer $(d>30 \mathrm{~nm})$, bulk transport starts to dominate the device current requiring higher bias for viable device current (space-charge-limited regime).

In conclusion, the $V_{b}$ and $T$ dependencies of our devices confirm valid GMR effects via injection and transport of spin-polarized carriers through the OSC spacer. We explained the carrier injection and transport into/in OSC and their impact on the GMR in OSC-based spin valves in terms of phonon-assisted tunneling and hopping in the presence of strong electric field. Observation of the spin precession will provide further proof for the spin transport in the OSC layers, which may require substantial improvement of material's mobility and interfacial quality.

\section{ACKNOWLEDGMENTS}

C.B.E. thanks M. S. Rzchowski for helpful discussions. This work was supported in part by the AFOSR under Grant No. FA9550-06-1-0175, DOE under Grants No. DE-FG0201ER45931, No. DE-FG02-86ER45271, No. DE-FG0206ER46327, NSF under Grant No. DMR-0805220, and ONR under Grant No. N00014-07-1-0215.
${ }^{1}$ A. Wolf, D. D. Awschalom, R. A. Buhrman, J. M. Daughton, S. von Molnár, M. L. Roukes, A. Y. Chtchelkanova, and D. M. Treger, Science 294, 1488 (2001).

${ }^{2}$ V. Dediu, M. Murgia, F. C. Matacotta, C. Taliani, and S. Barbanera, Solid State Commun. 122, 181 (2002).

${ }^{3}$ Z. H. Xiong, D. Wu, Z. V. Vardeny, and J. Shi, Nature (London) 427, 821 (2004).

${ }^{4}$ P. P. Ruden and D. L. Smith, J. Appl. Phys. 95, 4898 (2004).

${ }^{5}$ S. Majumdar, R. Laiho, P. Laukkanen, I. J. Väyrynen, H. S. Majumdar, and R. Österbacka, Appl. Phys. Lett. 89, 122114 (2006).

${ }^{6}$ S. Pramanik, C.-G. Stefanita, S. Patibandla, S. Bandyopadhyay, K. Garre, N. Harth, and M. Cahay, Nat. Nanotechnol. 2, 216 (2007).

${ }^{7}$ T. S. Santos, J. S. Lee, P. Migdal, I. C. Lekshmi, B. Satpati, and
J. S. Moodera, Phys. Rev. Lett. 98, 016601 (2007).

${ }^{8}$ J. H. Shim, K. V. Raman, Y. J. Park, T. S. Santos, G. X. Miao, B. Satpati, and J. S. Moodera, Phys. Rev. Lett. 100, 226603 (2008).

${ }^{9}$ W. Xu, G. J. Szulczewski, P. LeClair, I. Navarrete, R. Schad, G. Miao, H. Guo, and A. Gupta, Appl. Phys. Lett. 90, 072506 (2007).

${ }^{10}$ J. S. Jiang, J. E. Pearson, and S. D. Bader, Phys. Rev. B 77, 035303 (2008).

${ }^{11}$ H. Vinzelberg, J. Schumann, D. Elefant, R. B. Gangineni, J. Tomas, and B. Büchner, J. Appl. Phys. 103, 093720 (2008).

${ }^{12}$ T. Ikegami, I. Kawayama, M. Tonouchi, S. Nakao, Y. Yamashita, and H. Tada, Appl. Phys. Lett. 92, 153304 (2008).

${ }^{13}$ V. Dediu, L. E. Hueso, I. Bergenti, A. Riminucci, F. Borgatti, P. Graziosi, C. Newby, F. Casoli, M. P. De Jong, C. Taliani, and Y. Zhan, Phys. Rev. B 78, 115203 (2008). 
${ }^{14}$ A. J. Drew, J. Hoppler, L. Schulz, F. L. Pratt, P. Desai, P. Shakya, T. Kreouzis, W. P. Gillin, A. Suter, N. A. Morley, V. K. Malik, A. Dubroka, K. W. Kim, H. Bouyanfif, F. Bourqui, C. Bernhard, R. Scheuermann, G. J. Nieuwenhuys, T. Prokscha, and E. Morenzoni, Nature Mater. 8, 109 (2009).

${ }^{15}$ M. Cinchetti, K. Heimer, J.-P. Wustenberg, O. Andreyev, M. Bauer, S. Lach, C. Ziegler, Y. Gao, and M. Aeschlimann, Nature Mater. 8, 115 (2009).

${ }^{16}$ Y. Q. Zhan, M. P. de Jong, F. H. Li, V. Dediu, M. Fahlman, and W. R. Salaneck, Phys. Rev. B 78, 045208 (2008).

${ }^{17}$ Y. Liu, S. M. Watson, T. Lee, J. M. Gorham, H. E. Katz, J. A. Borchers, H. D. Fairbrother, and D. H. Reich, Phys. Rev. B 79, 075312 (2009).

${ }^{18}$ G. Szulczewski, S. Sanvito, and M. Coey, Nature Mater. 8, 693 (2009).

${ }^{19}$ M. Johnson and R. H. Silsbee, Phys. Rev. B 35, 4959 (1987).

${ }^{20}$ G. Schmidt, D. Ferrand, L. W. Molenkamp, A. T. Filip, and B. J. van Wees, Phys. Rev. B 62, R4790 (2000).

${ }^{21}$ E. I. Rashba, Phys. Rev. B 62, R16267 (2000).

${ }^{22}$ H. Yamada, Y. Ogawa, Y. Ishii, H. Sato, M. Kawasaki, H. Akoh, and Y. Tokura, Science 305, 646 (2004).

${ }^{23}$ H. Ishii, K. Sugiyama, E. Ito, and K. Seki, Adv. Mater. (Wein- heim, Ger.) 11, 605 (1999).

${ }^{24}$ J. J. Akerman, R. Escudero, C. Leighton, S. Kim, D. A. Rabson, Renu Whig Dave, J. M. Slaughter, and Ivan K. Schuller, J. Magn. Magn. Mater. 240, 86 (2002).

${ }^{25}$ J.-H. Park, E. Vescovo, H.-J. Kim, C. Kwon, R. Ramesh, and T. Venkatesan, Phys. Rev. Lett. 81, 1953 (1998).

${ }^{26}$ V. Garcia, M. Bibes, A. Barthélémy, M. Bowen, E. Jacquet, J.-P. Contour, and A. Fert, Phys. Rev. B 69, 052403 (2004).

${ }^{27}$ F. J. Wang, C. G. Yang, Z. V. Vardeny, and X. G. Li, Phys. Rev. B 75, 245324 (2007).

${ }^{28}$ S. Majumdar, H. Huhtinen, H. S. Majumdar, R. Laiho, and R. Österbacka, J. Appl. Phys. 104, 033910 (2008).

${ }^{29}$ T. S. Kim, Phys. Rev. B 72, 024401 (2005).

${ }^{30} \mathrm{~S}$. Mukhopadhyay and I. Das, Phys. Rev. Lett. 96, 026601 (2006).

${ }^{31}$ A. Kiveris, Š. Kudžmauskas, and P. Pipinys, Phys. Status Solidi A 37, 321 (1976).

${ }^{32}$ P. Pipinys and A. Kiveris, J. Phys.: Condens. Matter 17, 4147 (2005).

${ }^{33}$ P. A. Bobbert, W. Wagemans, F. W. A. van Oost, B. Koopmans, and M. Wohlgenannt, Phys. Rev. Lett. 102, 156604 (2009). 